\title{
FISIOTERAPIA LABORAL NA FORMAÇÃO ERGONÔMICA E CONSCIÊNCIA POSTURAL DE TRABALHADORES ADMINISTRATIVOS DE UMA INSTITUIÇÃO DE ENSINO SUPERIOR
}

\author{
Sara Cristina Freitas de Oliveira (1); \\ Neide Maria Gomes de Lucena (2) \\ María Carmen Villaverde Gutiérrez (3) \\ Paloma Lopes de Araújo Furtado (4) \\ Eliane Lourenço Rios (5) \\ José Juan Jiménez Moleón (6)
}

(1) Universidade de Granada, Doutoranda do Programa de Doctorado de Medicina Clínica y Salud Pública

e-mail: sarafta@hotmail.com

(2) Universidade Federal da Paraíba, Professora Dra do Departamento de Fisioterapia

e-mail: neidemariaglucena@hotmail.com

(3) Universidad de Granada, España, Professora Dra do Programa de Doctorado de Medicina Clínica y Salud Pública

e-mail: carmenvg@ugr.es

(4) Universidade Federal da Paraíba, Graduanda em Fisioterapia

e-mail: paloma_lops@hotmail.com

(5) Universidade Católica de Brasília, Graduanda em Fisioterapia

e-mail: eliane.rl3@gmail.com

(6)Universidad de Granada, España, Coordenador do Programa de Doctorado de Medicina Clínica y Salud Pública

e-mail:jjmoleon@ugr.es

\begin{abstract}
RESUMO
O objetivo deste estudo foi avaliar a repercussão de um Programa de Fisioterapia Laboral (PFISLAB) na formação ergonômica e consciência postural de trabalhadores administrativos de uma instituição de ensino superior. Trata-se de um estudo de caso descritivo com abordagem quantitativa. Após seis meses do término do PFISLAB, 8 trabalhadores foram entrevistados e afirmaram conhecer a postura adequada no trabalho; $87,5 \%$ passaram a adotar os ajustes ergonômicos, $50 \%$ referiram que ainda seguiam sentido os efeitos positivos. Todos os participantes confirmaram contribuição do programa na conscientização da postura correta no trabalho e aumento do conhecimento em ergonomia.
\end{abstract}




\section{ABSTRACT}

The aim of this study was to evaluate the effect of an occupational physical therapy program on ergonomic training and on postural awareness among a administrative workers of an institution of higher education. This is a descriptive case studywith a quantitative approach. After six months of intervention, 8 workers were interviewed and claimed to know the proper posture at work; $87.5 \%$ went on to adopt ergonomic adjustments, $50 \%$ reported to keep on feeling the positive effects. All participants confirmed the effectiveness of the program to raise awareness of workplace posture. They also increased their knowledge related to the field of ergonomics.

\section{INTRODUÇÃO}

A fisioterapia laboral é uma especialidade da fisioterapia que pode atuar dentro e fora das empresas ou organizações, visando a prevenção, promoção e reabilitação da saúde do trabalhador. O Fisioterapeuta desempenha papel importante dentro de uma equipe multidisciplinar de saúde ocupacional, pois está apto a desenvolver e executar estratégias e programas de intervenção ergonômica, em especial dirigidas ao controle de distúrbios musculoesqueléticos e de dores na coluna vertebral ao promover a formação ergonômica e a higiene postural dos trabalhadores, a análise e adaptação dos postos de trabalho e a melhora do desempenho morfofuncional no trabalho através da cinesioterapia individual ou coletiva (COFFITO, 2011; FIGL-HERTLEIN et al., 2014).

A formação ergonômica é uma estratégia que busca a educação dos trabalhadores sobre posturas e métodos de trabalho com uso mais adequado da mecânica corporal. Proporciona conhecimento sobre o uso adequado do mobiliário e dos instrumentos de trabalho e sobre os distúrbios musculoesqueléticos. Capacita e incentiva o indivíduo a mudanças comportamentais e ao uso de gestos laborais mais eficientes através de atividades práticas e orientações aplicadas diretamente às situações do seu posto de trabalho ou utilizando recursos tradicionais como palestras e panfletos informativos (DRIESSEN et al., 2011; MAHMUD NORASHIKIN; KENNY; RAHMAN, 2012).

A ergonomia física intervém diretamente sobre o posto de trabalho modificando e/ou ajustando o mobiliário, instrumentos e acessórios, de forma que respeitem as medidas antropométricas, sejam funcionais e desta forma evitem ou aliviem a sobrecarga biomecânica sobre o corpo do trabalhador (CHOOBINEH et al., 2011; DRIESSEN et al., 2011).

O trabalhador administrativo desenvolve suas atividades laborais na postura prolongada sentada diante do computador(especialmente em ambiente ergonômicos inadequados) e sua relação com surgimento de dores na coluna é discutida em diversas pesquisas. Funções que exigem o uso de teclado e monitor de vídeo muitas vezes expõem estes trabalhadores a posturas inadequadas e os movimentos repetitivos e constantes das extremidades superiores têm sido frequentemente relacionados como causas de dor em ombro e pescoço. De forma que os Distúrbios Osteomusculares Relacionados ao Trabalho (DORT) são comumente relatados por trabalhadores administrativos. (FABRIZIO, 2009; MAHMUD NORASHIKIN; KENNY; RAHMAN, 2012; NEJATI et al., 2014).

Essas disfunções são frequentes e crescentes nos postos de trabalho, portanto, é necessária a implantação de métodos preventivos contra esses distúrbios como adaptação ergonômica, ginástica laboral e outras estratégias que foquem a saúde do trabalhador. Desta forma é possível evitar ou diminuir o absenteísmo, reduzir custos e melhorar o 
rendimento da empresa. (ALCÂNTARA; NUNES; FERREIRA, 2011; MEHRPARVAR et al., 2014; VIVIAN R. SAKAMOTO; NAVARRO, 2011).A fisioterapia laboral lança mão da cinesiologia para direcionar e aplicar programas de exercícios cinesioterapêuticos especificamente dirigidos ao controle de lesões associadas à carga física de trabalho, uma vez que esta ciência tem como objeto de estudo o movimento corporal humano, o que leva estes profissionais a potencializar a capacidade do individuo de realizar movimentos e assim melhorar o desempenho e a eficiência do trabalhador, com a intenção de prevenir e/ou reduzir o surgimento de patologias osteomusculares relacionadas com o trabalho(ALLEN et al., 2015; NOGUEIRA; NAVEGA, 2011; VÉLEZ et al., 2011).

Em razão disso o objetivo deste trabalho foi avaliar a repercussão de um programa de fisioterapia laboral sobre a formação ergonômica e postural de trabalhadores administrativos de uma instituição de ensino superior.

\section{MÉTODO}

Estudo de caso do tipo descritivo com abordagem quantitativa. Trata-se de uma vertente de um trabalho de tese doutoral em andamento (OLIVEIRA,2015) e foi aprovada pelo Comitê de Ética e Pesquisa da Universidade Católica de Brasília. Todos os trabalhadores administrativos da instituição foram convidados a participar através de e-mail.

Os critérios de inclusão foram: trabalhar predominantemente na postura sentada, usar o computador como principal instrumento de trabalho e apresentarem sintomas musculoesqueléticos (dor, dormência ou desconforto) nos últimos 12 meses. Foram excluídos sujeitos com lesões osteomusculares ou neurológicas graves, deficiência cognitiva ou sensório motora e grávidas. A seleção foi aleatória com amostra final de 8 participantes que participaram do programa de intervenção, cumpriram $75 \%$ das sessões, que permaneceram na empresa após seis meses do término do programa e que assinaram o Termo de Consentimento Livre e Esclarecido.

O Programa de Fisioterapia Laboral (PFISLAB)foi desenvolvido no primeiro semestre de 2015 na própria instituição e constou de adaptação ergonômica, orientações sobre higiene postural (educação ergonômica e postural sobre posturas adequadas dentro e fora do trabalho) e cinesioterapia em grupo. As sessões de cinesioterapia foram realizadas duas vezes por semana durante 30 minutos por 6 meses, depois de suas atividades laborais, mas dentro do horário regular de trabalho.

Foi fundamentada nos métodos de Reeducação Postural Global (RPG) (SOUCHARD, 2007) e nos princípios do Pilates no solo (CRAIG, 2005), objetivando promover a consciência postural, estabilidade ao complexo muscular da coluna vertebral e dos membros superiores e melhorar a postura.

Os ajustes ergonômicos dos postos dos trabalhadores que participaram do programa seguiram as recomendações do Check-list para avaliação das condições ergonômicas em postos de trabalho e ambientes informatizados de Couto (2014). Foram realizadas adaptações adequadas do posicionamento do mobiliário (assento e mesa) e de seus principais instrumentos de trabalho (computador, monitor, mouse e teclado).

Durante o desenvolvimento do programas e administrou atividades educativas teóricopráticas com os seguintes temas: mecanismos produtores da dor na coluna vertebral e dos Distúrbios Osteomusculares Relacionados ao Trabalho (DORT); noções de anatomia e biomecânica da coluna vertebral; informações ergonômicas sobre posturas adequadas nos postos de trabalho com computador; posição ideal domobiliário e dos instrumentos de trabalho além de estimular hábitos saudáveis no trabalho e na vida diária para a prevenção dos DORT e da dor na coluna. 
A coleta de dados foi realizada 6 meses após o término do PFISLAB, como medida seguimento, objetivando verificar se houve permanência dos conhecimentos e efeitos do referido programa quanto à formação ergonômica e consciência postural dos participantes envolvidos no estudo, para tanto, utilizou-se de um "Questionário para avaliação da percepção dos trabalhadores sobre o Programa de Fisioterapia Laboral (PFISLAB)", elaborado a partir do Questionnaire Awareness of WMSD-Related Knowledge de Shuai et al (2014). No mesmo questionário o trabalhador tinha opções binárias de resposta (sim ou não) de acordo com a sua percepção de antes e depois de participar do programa.Por exemplo,"Antes do PFISLAB você sabia o que são os DORTs?E após o PFISLAB? Em caso positivo o que você entende por DORT?". Este questionário foi aplicado através de entrevistas utilizando-se de um espaço adequado (uma sala da empresa) no horário regular de trabalho, sem qualquer ônus para o trabalhador.

Foi realizada análise estatística descritiva dos dados (média, desvio padrão, frequências absolutas e relativas) com auxílio do programa SPSS 23.

\section{RESULTADOS}

Foram entrevistados 8 trabalhadores (5 mulheres e 3 homens) com idade média de 38 anos $( \pm 9,1)$, mínima de 29 anos e máxima de 51 anos; $75 \%(n=6)$ afirmaram estar a menos de 6 anos na função; $87,5 \%(n=7)$ relataram ter jornada diária de trabalho de 8 ou mais horas.

Os resultados das tabelas 1 e 2 são apresentados de acordo com a percepção dos participantes antes e adquiridos depois de seis meses do término do PFISLAB no tocante a: conhecimentos de saúde ocupacional e ergonomia.

A Percepção dos trabalhadores sobre os conhecimentos de saúde ocupacional e ergonomia e postura adotada durante o trabalho com o computador antes e adquiridos depois de seis meses do término do PFISLAB, pode ser verificada na tabela 1.

Tabela 1 - Percepção dos trabalhadores sobre os conhecimentos de saúde ocupacional e ergonomia antes e adquiridos após de seis meses do término do PFISLAB

\begin{tabular}{|c|c|c|c|c|}
\hline $\mathrm{N}=8$ & & $\%$ & $\begin{array}{l}\text { Af } \\
\mathrm{PF}\end{array}$ & $\begin{array}{l}\text { ESES DO } \\
\%\end{array}$ \\
\hline $\begin{array}{l}\text { Conhecimento sobre } \\
\text { a definição de DORT }\end{array}$ & 2 & 25,0 & 8 & 100 \\
\hline $\begin{array}{l}\text { Conhecimento sobre } \\
\text { prevenção de DORT }\end{array}$ & 3 & 37,5 & 8 & 100 \\
\hline
\end{tabular}




\begin{tabular}{|l|ll|ll|}
\hline $\begin{array}{l}\text { Conhecimento das } \\
\text { curvas normais da } \\
\text { coluna vertebral }\end{array}$ & 3 & 37,5 & 8 & 100 \\
\hline $\begin{array}{l}\text { Conhecimento da } \\
\text { postura adequada no } \\
\text { uso do computador }\end{array}$ & 2 & 25,0 & 8 & 100 \\
\hline $\begin{array}{l}\text { Conhecimento do } \\
\text { ajuste do assento }\end{array}$ & 1 & 12,5 & 8 & 100 \\
\hline
\end{tabular}

A Tabela 2 apresenta a percepção dos trabalhadores sobre a postura adotada no uso do computadorantes e adquiridos depois de seis meses do término do PFISLAB.

Tabela 2 - Percepção dos trabalhadores sobre postura adotada no uso do computador antes e adquiridos depois de seis meses do término do PFISLAB

\begin{tabular}{|l|rr|rr|}
\hline \multicolumn{1}{|c|}{$\mathbf{N = 8}$} & \multicolumn{2}{|c|}{$\begin{array}{c}\text { ANTES DO } \\
\text { PFISLAB }\end{array}$} & \multicolumn{2}{c|}{$\begin{array}{c}\text { APÓS 6 MESES } \\
\text { DO PFISLAB }\end{array}$} \\
& $\mathbf{n}$ & $\%$ & $\mathbf{n}$ & $\%$ \\
\hline $\begin{array}{l}\text { Mesa/monitor/assento } \\
\text { alto }\end{array}$ & 1 & 12,5 & 0 & 0 \\
\hline $\begin{array}{l}\text { Mesa/monitor/assento } \\
\text { baixo }\end{array}$ & 6 & 75,0 & 1 & 12,5 \\
\hline & & & & \\
\hline $\begin{array}{l}\text { Mesa/monitor/assento } \\
\text { altura adequada }\end{array}$ & 1 & 12,5 & 7 & 87,5 \\
\hline
\end{tabular}


Os resultados das tabelas 3 e 4 são apresentados segundo a percepção dos participantes depois de seis meses do término do PFISLAB no que se refere a: duração dos efeitos positivos do programa e contribuição do mesmo na melhora da consciência postural.

Tabela 3 - Percepção dos trabalhadores sobre duração dos efeitos positivos do PFISLAB após seis meses do término do PFISLAB

\begin{tabular}{|l|ll|}
\hline \multicolumn{1}{|c|}{$\mathbf{N = 8}$} & $\mathbf{n}$ & $\mathbf{\%}$ \\
\hline Uma semana & 1 & 12,5 \\
\hline 30 dias & 1 & 12,5 \\
\hline 6 meses & 1 & 25,0 \\
\hline $\begin{array}{l}\text { Segue sentindo os } \\
\text { efeitos }\end{array}$ & 4 & 50,0 \\
\hline
\end{tabular}

Tabela 4 - Percepção dos trabalhadores sobre contribuição do PFISLAB

\begin{tabular}{|l|lr|}
\hline \multicolumn{1}{|c|}{ N=8 } & $\mathbf{n}$ & $\%$ \\
\hline Atenção à postura no trabalho após o PFISLAB & 7 & 87,5 \\
\hline $\begin{array}{l}\text { Contribuição do PFISLAB para melhora da postura no } \\
\text { trabalho }\end{array}$ & 8 & 100 \\
\hline $\begin{array}{l}\text { Contribuição do PFISLABna prevenção e redução dos } \\
\text { sintomas }\end{array}$ & 8 & 100 \\
\hline Recomenda o PFISLAB a outros trabalhadores & 8 & 100 \\
\hline
\end{tabular}

A tabelas 5 apresenta os resultados segundo a percepção dos participantes após seis meses do término do PFISLAB no que se refere à estratégia ideal para a prevenção de DORT e dores na coluna vertebral. 
Tabela 5- Percepção dos trabalhadores sobre qual a estratégia ideal para a prevenção de DORT e dores na coluna vertebral.

\begin{tabular}{|l|ll|}
\hline \multicolumn{1}{|c|}{$\mathbf{N = 8}$} & $\mathbf{n}$ & $\%$ \\
\hline $\begin{array}{l}\text { Orientação e adaptação ergonômica dos postos de } \\
\text { trabalho }\end{array}$ & 0 & 0 \\
\hline Cinesioterapia & 0 & 0 \\
\hline $\begin{array}{l}\text { Associação entre orientação, adaptação ergonômica do } \\
\text { posto de trabalho e cinesioterapia }\end{array}$ & 8 & 100 \\
\hline
\end{tabular}

\section{DISCUSSÃO}

Este trabalho buscou avaliar a percepção de um grupo de trabalhadores administrativos de uma instituição de ensino superior submetidos a um programa de fisioterapia laboral (PFISLAB)que envolveu adaptações ergonômicas dos postos de trabalho, formação em ergonomia e cinesioterapia, seis meses após o término do programa.

Os resultados deste trabalho convergem com diversos outros estudos que têm demonstrado que a educação ergonômica leva a mudanças comportamentais no ambiente de trabalho com eficácia na prevenção dos DORT, proporcionando melhora de sintomas osteomusculares como dor e desconforto de trabalhadores comparados a outros que atuam no mesmo ambiente de trabalho, mas que não receberam este tipo de formação(MAHMUD NORASHIKIN; KENNY; RAHMAN, 2012; SHUAI et al., 2014).

Em outra pesquisa que buscou verificar os efeitos da educação ergonômica em trabalhadores de escritório, utilizou de um questionário para investigar os hábitos adequados e inadequados na estação de trabalhorelacionados com o uso do computador, teclado, mouse, cadeira e mesa. Após 6 meses de intervenção,a formação e ajustes ergonômicos proporcionaram melhora nos hábitos dos trabalhadores no ambiente de trabalho além da redução dos distúrbios musculoesqueléticos relatados (MAHMUD et al., 2011).

Um estudo longitudinal realizado entre professores na China apontou os efeitos que um programa de educação ergonômica pode ter sobre a consciência e na mudanças de hábitos posturaisdos trabalhadores. Implicando em mudança significativa no comportamento saudável e no uso de postura adequadas no ambiente de trabalhoem 6 meses após a intervenção. Os conhecimentos relacionados aos DORTs fisiopatologia, biomecânica e fatores de prevenção aumentaram: sobre definiçãode DORTs (de 25,4\%/n=89 para $47,5 \% / n=156)$,sobre prevenção de DORT $(70,0 \% / n=245$ para $79,5 \% / n=261)$ esobre curvas fisiológicas da coluna ( de $20,29 \% / n=71$ para $55.4 \% / n=182$ ). Também cresceram em conhecimento em relação a fatores mais ergonômicos como ajuste ideal da altura do assento (de $20,8 \% / \mathrm{n}=73$ para $32,6 \% / \mathrm{n}=107$ ) e a postura correta na mesa de trabalho (de $46,5 \% / n=163$ para de $73,1 \% / n=240)$. O comportamento e atitude dos professores para a prevenção dos DORTs persistiu com êxito durante pelo menos 12 mesesde intervenção, quando também foi verificadouma queda significativa na prevalência de dores ou distúrbios relatados pelos professores(SHUAl et al., 2014), dados estes que corroboram com a pesquisa em pauta. 
No presente estudo, segundo a percepção dos trabalhadores, os conhecimentos de saúde ocupacional e ergonomia adquiridos depois de seis meses do término do PFISLAB também aumentaram. Por exemplo: saber definir DORT; sobre os DORTs serem uma enfermidade possível de ser prevenida ou controlada. Bem como qual a postura adequada ao se trabalhar com o computador. Quase todos os participantes passaram adotar os ajustes adequados do conjunto mesa/monitor/assento após o PFISLAB.

Com relação ao tempo de duração dos efeitos do programa, a maioria afirmou ainda sentir os efeitos positivos da intervenção após seis meses do seu término. Quase todos relataram que após o PFISLAB passaram a ter atenção especial com a postura no trabalho. Estes resultados corroboram com o estudo (VÉLEZ et al., 2011), que relataa experiência de um programa de fisioterapia laboral em trabalhadores de uma empresa de produção de geotêxteis para a construção na Colômbia utilizou de técnicas de cinesioterapia que visavam proporcionar estabilidade ao complexo muscular da coluna vertebral, evitar lesões osteomusculares e melhorar a postura para a prevenção de dor lombar sobre uma amostra de 48 colaboradores do gênero masculino a qual também se aplicou uma enquete de percepção sobre o programa à população assistente. Após doze meses de intervenção o $94 \%$ da população reportou diminuição da fadiga muscular no nível lombar e $96 \%$ conseguiu relatou melhora no desempenho de suas atividades laborais.

Os resultados obtidos ao final deste estudo corroboram com (ALLEN et al., 2015) que afirma que os fisioterapeutas tem experiência avaliando as forças mecânicas no trabalho sobre corpo humano e preparando o corpo para a carga de trabalho enquanto que a ergonomia centra-se no ambiente de trabalho, assegurando que as exigências do trabalho correspondam às capacidades físicas do trabalhador. As condições do local de trabalho podem ser reestruturadas ou modificadas para reduzir o estresse que causam os distúrbios músculoesqueléticos. As lesões geralmente ocorrem quando forças biomecânicas excedem as limitações das estruturas do aparelho locomotor (músculos, tendões, articulações, etc).

$\mathrm{Na}$ presente pesquisa, quando questionados sobre qual estratégia os trabalhadores consideravam ideal para prevenção de DORT e dores na coluna vertebral,todos concordaram que seria a associação entre orientação, ajustes ergonômicos do posto de trabalho assim como as sessões de cinesioterapia desenvolvidas. Todos os trabalhadores apontaram a contribuição do programa (PFISLAB) na prevenção e redução dos sintomas osteomusculares e indicariam o referido programa como uma atividade permanente na instituição estudada, fazendo parte de forma regular da equipe de saúde e medicina do trabalho da empresa.

\section{CONCLUSÃO}

Os resultados demonstraram que os trabalhadores avaliados obtiveram crescimento em educação ergonômica e postural e demonstraram de forma concreta a formação ergonômica adquirida.Também pôde ser observado que os participantes do PFISLAB consideram de extrema importância a ação da Fisioterapia Laboral no desenvolvimento da consciência ergonômica da empresa e entre seus trabalhadores. Associar a adaptação e a formação ergonômica dos trabalhadores a exercícios terapêuticos que preparem seu corpo para a carga de trabalho parece ser a melhor estratégia de intervenção na prevenção de distúrbios musculoesqueléticos e de dores na coluna vertebral relacionadas com o trabalho.

\section{REFERÊNCIAS BIBLIOGRÁFICAS}

ALCÂNTARA, Marcus Alessandro; NUNES, Gabriela Da Silveira; FERREIRA, Bárbara Coura Moreira Dos Santos. Distúrbios Osteomusculares Relacionados Ao Trabalho: O Perfil sos Trabalhadores em Benefício Previdenciário em Diamantina (MG, Brasil). Ciência \& Saúde Coletiva, V. 16, N. 8, P. 3427-3436, 2011. 
ALLEN, Steve Et Al. Prevention-Know Your Risks. Therapeutic Outlook, V. 9, N. 2, P. 8-11, 2015. Disponível Em: <Https://Www.Therapeuticassociates.Com/Therapeutic-Outlook-Magazine/>. Acesso Em: 20 Jun. 2016.

CHOOBINEH, Alireza Et Al. The Impact of Ergonomics Intervention on Psychosocial Factors and Musculoskeletal Symptoms Among Office Workers. International Journal Of Industrial Ergonomics, V. 41, N. 6, P. 671-676, 2011. Disponível Em: <Http://Dx.Doi.Org/10.1016/J.Ergon.2011.08.007>.

COFFITO, Conselho Federal De Fisioterapia E Terapia Ocupacional. Disciplina a Especialidade Profissional de Fisioterapia Do Trabalho e dá Outras Providências. Brasil: COFFITO, Conselho Federal De Fisioterapia E Terapia Ocupacional. Disponível Em:

<Http://Www.Coffito.Org.Br/Site/Index.Php/Home/Resolucoes-Coffito/482-Resolucao-N-403-2011Disciplina-A-Especialidade-Profissional-De-Fisioterapia-Do-Trabalho-E-Da-Outras-

Providencias.Html>. , 2011.

COUTO, H.A. Check-List para Avaliação das Condições Ergonômicas em Postos de Trabalho e Ambientes Informatizados. Disponível Em: <Http://Www.Ergoltda.Com.Br/Checklist/Index.Html>. Acesso Em: 2 Feb. 2015.

CRAIG, C. Pilates com Bola. São Paulo, Brasil: Editora Phorte, 2005.

DRIESSEN, Maurice T. Et Al. The Effectiveness Of Participatory Ergonomics To Prevent LowBack and Neck Pain - Results Of A Cluster Randomized Controlled Trial. Scandinavian Journal Of Work, Environment And Health, V. 37, N. 5, P. 383-393, 2011.

FABRIZIO, P. Ergonomic Intervention in the Treatment of a Patient with Upper Extremity and Neck Pain. Physical Therapy, V. 89, N. 4, P. 351-360, 2009.

FIGL-HERTLEIN, A. Et Al. A Physiotherapy-Directed Occupational Health Programme for Austrian School Teachers: A Cluster Randomised Pilot Study. Physiotherapy (United Kingdom), V. 100, N. 1, P. 20-26, 2014.

MAHMUD, Norashikin Et Al. Ergonomic Training Reduces Musculoskeletal Disorders Among Ofice Workers : Results From the 6-Month Follow-Up. V. 18, N. 26, P. 16-26, 2011.

MAHMUD NORASHIKIN; KENNY, Dianna T; RAHMAN, Hamidah Ab. The Effect of Workplace Office Ergonomics Intervention on Reducing Neck and Shoulder Complaints and Sickness Absence. International Proceedings of Economics Development \& Research, V. 42, N. JANUARY 2011, P. 186-189, 2012.

MEHRPARVAR, Amir Houshang Et Al. Ergonomic Intervention, Workplace Exercises and Musculoskeletal Complaints: A Comparative Study. Medical Journal Of The Islamic Republic Of Iran, V. 28, P. 69, 2014. Disponível Em:

$<$ Http://Www.Pubmedcentral.Nih.Gov/Articlerender.Fcgi?Artid=4219902\&Tool=Pmcentrez\&Rendertyp e=Abstract>.

NEJATI, Parisa Et Al. The Relationship of Forward Head Posture and Rounded Shoulders With Neck Pain in Iranian Office Workers. Medical Journal Of The Islamic Republic Of Iran, V. 28, P. 26, 2014. Disponível Em:

$<$ Http://Www.Pubmedcentral.Nih.Gov/Articlerender.Fcgi?Artid=4154278\&Tool=Pmcentrez\&Rendertyp e=Abstract>

NOGUEIRA, Helen Cristina; NAVEGA, Marcelo Tavella. Influência Da Escola De Postura Na Qualidade de Vida, Capacidade Funcional, Intensidade De Dor E Flexibilidade De Trabalhadores Administrativos. Fisioterapia E Pesquisa, V. 18, N. 4, P. 353-358, 2011.

SHUAI, Jian Et Al. Assessing the Effects of an Educational Program for the Prevention of WorkRelated Musculoskeletal Disorders Among School Teachers. BMC Public Health, V. 14, N. 1, P. 1211, 2014. Disponível Em:

<Http://Www.Pubmedcentral.Nih.Gov/Articlerender.Fcgi?Artid=4256741\&Tool=Pmcentrez\&Rendertyp 
$\mathrm{e}=$ Abstract $>$.

SOUCHARD, P. Autoposturas da RPG. São Paulo, Brasil: E. Realizações, 2007.

VÉLEZ, Claudia Lucia Et Al. Implementación de "Escuela De Espalda" mediante la Técnica de "Core" en una Fábrica de Geotextiles para la Prevención del Dolor Lumbar. Revista Ciencias De La Salud, V. 9, N. 1, P. 57-71, 2011.

VIVIAN R. SAKAMOTO, Felipe F. Donatto; NAVARRO, Antonio Coppi. A Influência da Ginastica Laboral e Da Qualidade Alimentar Empresarial na Sintomatologia do Quadro de Dor em Funcionários do Setor Administrativo. Revista Brasileira de Nutrição Esportiva, v. 5, p. 62-69, 2011. 